\title{
TTR
}

Traduction, terminologie, rédaction

\section{Michelle Woods. Censoring Translation: Censorship, Theatre, and the Politics of Translation. Londres et New York, Continuum, 2012, 175 p.}

\section{Nicole Nolette}

Volume 26, numéro 2, 2e semestre 2013

URI : https://id.erudit.org/iderudit/1037143ar

DOI : https://doi.org/10.7202/1037143ar

Aller au sommaire du numéro

Éditeur(s)

Association canadienne de traductologie

ISSN

0835-8443 (imprimé)

1708-2188 (numérique)

Découvrir la revue

Citer ce compte rendu

Nolette, N. (2013). Compte rendu de [Michelle Woods. Censoring Translation: Censorship, Theatre, and the Politics of Translation. Londres et New York,

Continuum, 2012, 175 p.] TTR, 26(2), 281-284. https://doi.org/10.7202/1037143ar d'utilisation que vous pouvez consulter en ligne.

https://apropos.erudit.org/fr/usagers/politique-dutilisation/ 
ou moins locales/globales? Quelles sont les limites et difficultés du passage d'un niveau (ou d'un ordre) d'analyse à l'autre? On peut regretter que l'auteur n'aborde pas directement ces questions. Cela dit, en tentant, à plusieurs reprises, de «traduire» en termes «luhmanniens » des études de cas traductologiques, il offre à chacun la possibilité de se forger une idée. Ainsi chaque lecteur pourra juger, par lui-même, de ce travail de «traduction». Ceux et celles qui, toute bigoterie mise à part, préfèrent expliquer la traduction en mobilisant les notions de sujet ou d'agent (plutôt qu'en invoquant la rationalité d'un système) seront sans doute mitigé(e)s tandis que les traductologues ayant un goût prononcé pour l'abstraction pure et qui sont en quête d'une théorie unificatrice à prétention universelle seront sûrement séduits. Au final, tous apprécieront le travail conceptuel, la richesse des idées, l'érudition, la rigueur, l'ambition et les analogies audacieuses qui font de cet essai résultat d'une réflexion aussi profonde qu'originale - un livre exigeant et une contribution importante à la sociologie de la traduction.

\section{Références}

Ferrarese, Estelle (2012). «À propos de Niklas Luhmann, Systèmes sociaux. Esquisse d'une théorie générale». Raison publique, 14 septembre 2012. [http://www.raison-publique.fr/auteur1445. html.]

Folkart, Barbara (1991). Le conflit des énonciations: traduction et discours rapporté. Québec, Les Éditions Balzac, «l'Univers des discours».

Heilbron, Johan (1999). «Toward a Sociology of Translation: Book Translations as a Cultural World-System». European Journal of Social Theory, 2, 4, p. 429-444.

HÉLÈne BuzeLIN Université de Montréal

Michelle Woods. Censoring Translation: Censorship, Theatre, and the Politics of Translation. Londres et New York, Continuum, 2012, 175 p.

Censoring Translation: Censorship, Theatre, and the Politics of Translation est le deuxième ouvrage publié par Michelle Woods, professeure agrégée au campus New Paltz de la State University of New York. Elle y poursuit une réflexion amorcée dans son premier ouvrage, Translating Milan Kundera, autour de la littérature 
tchèque, sa traduction et sa censure. Dans Censoring Translation, l'analyse de Woods porte sur les pièces de théâtre de Václav Havel - aussi connu comme président de la République fédérale tchèque et slovaque de 1989 à 1992, puis de la République tchèque de 1993 à 2003 - et leur traduction vers l'anglais. Elle suggère que les pièces de Havel subissent une double censure: celle imposée par le régime communiste tchécoslovaque et celle imposée par le monde anglo-américain aux pièces en traduction.

Woods file un argument tout en nuances autour de la censure et de l'œuvre théâtrale de Havel, en commençant par l'inscription de la censure à même l'écriture de cet auteur dramatique. Selon elle, cette écriture déploie une interrogation existentielle sur la censure, une réaction artistique à celle-ci plutôt qu'une attaque politique directe. Les pièces de Havel interpellent les spectateurs par une poétique particulière alliant style prolixe, répétitions et absurdismes, une poétique qui ne vise pas l'allégorie du style de communication du régime communiste, mais qui critique la construction de toute réalité par le langage. Woods, suivant la traductologue autrichienne Mandana Taban, nomme «analogical translation" cette réaction artistique aux conditions de la censure, "a mode of translation that is not an encoding of secret political messages as subtext but that secretly translates the conditions of censorship into an artistic text in order to investigate and explore the modality of censorship» (p. 41).

Elle continue son exploration de la censure en étudiant ce qu'on a le plus souvent tendance à associer à cette dernière, c'està-dire les pratiques d'un régime totalitaire. En Tchécoslovaquie, ces pratiques, assurées par une agence d'État littéraire et théâtrale, Divadelni, literárni, audovizuálni agentura (DILIA), se dirigeaient non pas vers l'interférence textuelle, comme on aurait pu s'y attendre, mais vers la diffusion des textes (contrats et salaires révoqués, retards dans la bureaucratie). Woods éclaire admirablement l'ambivalence autour de ces pratiques: d'un côté, l'agence compliquait la libre circulation de l'œuvre de Havel; de l'autre, elle souhaitait ardemment faire rayonner les traductions vers l'anglais au Royaume-Uni et aux États-Unis afin d'augmenter le prestige et le revenu du régime. Autre paradoxe: la DILIA n'interférait pas dans les textes de Havel (même si elle y interdisait l'accès en Tchécoslovaquie), alors que les intervenants des milieux théâtraux au Royaume-Uni et aux États-Unis exerçaient 
constamment des pressions pour que les traductions de ces textes soient retranchées ou adaptées. Le récit que construit Woods autour des traductions de l'œuvre de Havel pour les scènes anglophones renverse habilement l'idée reçue qui situe la censure dans les pays de l'Est en lui opposant la liberté d'expression des pays de l'Ouest. Elle place ainsi l'Ouest devant ses propres mécanismes de «covert censorship» (p. 41) et de "market censorship» (p. 124).

La critique de Woods à l'égard de l'Ouest est sévère: on s'est approprié l'œuvre théâtrale de Havel en la réduisant à un objet politique pour lequel on pourrait vendre des billets de théâtre. En outre, les productions américaines du théâtre de Havel misaient sur celui-ci comme objet de censure en Tchécoslovaquie pour entériner la dichotomie simpliste faisant de l'Ouest le lieu de la liberté et l'Est celui de la répression. De surcroît, en conformité avec les lois du marché, la censure tacite du monde anglo-américain s'est effectuée à des fins économiques plutôt qu'idéologiques: « censored theatre, seditious elsewhere, is sexy and it sells» (p. 131). Woods déplie toutes les couches de cette marchandisation de l'Est censuré dans les spectacles de Havel en traduction en citant des programmes de spectacles, des témoignages de metteurs en scène et de producteurs de théâtre, des critiques américains et une correspondance abondante entre Havel et sa traductrice, Vera Blackwell. On voit bien l'incompréhension entre, d'un côté, l'auteur et la traductrice tchèques, et de l'autre, les producteurs de théâtre, metteurs en scène et critiques anglo-américains. Les premiers tentaient de faire comprendre aux derniers que plus on insistait sur le courage politique de Havel (ce qui vendait des billets de théâtre dans l'Ouest, mais qui ne transparaît pas dans le style de l'auteur), plus on mettait sa vie en péril en Tchécoslovaquie (ce qui nétait pas encore le cas avant la mise en scène anglo-américaine des pièces traduites en anglais). L'étude de cas des traductions théâtrales de Havel offre ainsi un aperçu saisissant de la dialectique de la guerre froide. Elle incite tout autant à réfléchir sur notre compréhension de la censure et sur comment celle-ci a été conditionnée par les rapports Est-Ouest issus de cette guerre.

Aux deux grandes formes de censure externes à l'œuvre (censure politique en Tchécoslovaquie et censure économique, axée sur les lois du marché, en Angleterre et aux États-Unis), Woods en ajoute une troisième: la censure (du rôle) de la traductrice, Vera Blackwell, dans le monde peuplé d'hommes quétait le théâtre 
américain et anglais des années 1960 à 1990. En plus de souligner une forme de censure sexiste à laquelle Blackwell a dû faire face, Woods a le mérite d'illuminer une situation particulière à la traduction théâtrale: on demande souvent à une femme de la même origine que l'auteur du texte d'effectuer une première traduction «littérale», puis à un homme, souvent un dramaturge mieux établi, d'y inscrire un style théâtral ou une certaine «jouabilité». Woods montre que pour Blackwell, la traduction nétait pas un travail littéral, mais bien littéraire, et que les modifications imposées par les dramaturges des cultures d'arrivée, souvent justifiées sous l'enseigne de la jouabilité, menaient à une destruction systématique de la lettre de l'œuvre de Havel.

À force de lire Woods, on en vient à voir des formes de censure de la traduction partout, même dans le texte de Woods lui-même. En effet, on se demande pourquoi l'auteure n'a pas inclus d'analyse des traductions de l'œuvre de Havel ou d'explication du choix de Blackwell et Havel, deux individus d'origine tchécoslovaque, d'échanger en anglais dans leur correspondance amplement citée. On ne trouve que deux traces de Woods comme traductrice du tchèque (p.110 et p. 165) dans Censoring Translation, une invisibilité flagrante à la lumière des propos sur la censure tacite. Un même manque de transparence marque la structure de l'ouvrage, ce qui masque l'absence d'une ligne directrice claire. En effet, avec sa préface, sa très longue introduction et ses trois chapitres sur la censure et les contraintes, sur la censure du genre et sur la censure du marché, l'organisation de l'ouvrage fait en sorte qu'on tourne trois fois autour de la même étude de cas et qu'on en sort un peu étourdi.

En revanche, on en sort aussi vivement intéressé par le sujet, avec une conception légèrement étendue de la censure et, surtout, avec une connaissance détaillée de l'œuvre théâtrale de Václav Havel, de sa traduction vers l'anglais et des formes de censure qui la traversent.

Nicole Nolette
Université McGill 